\title{
UNIVERSAL HEALTH COVERAGE
}

Universal Health Coverage (UHC) refers to the scenario wherein all the individuals and sections of society irrespective of any socio-demographic attributes are able to access the health care services without being subjected to any economic hardships. In addition, it ensures that the quality of the spectrum of offered health care services (ranging from health promotion, protection, therapeutic, rehabilitation, etc.) is of such standard to ameliorate the health standards of the people who are beneficiaries of the services.

The available estimates have revealed that close to $50 \%$ of the global population are still deprived of accessing essential health care and millions of people are being pushed into poverty due to catastrophic out-of-pocket expenditures on the health care. Acknowledging the magnitude of the problem and the indispensable need to take prompt action, the year 2030 has been fixed to accomplish universal health coverage. Moreover, UHC has been identified as an essential component of the Sustainable Development Goals (SDGs) and a lot needs to be done to stay on track to accomplish the same.

It is encouraging that some of the nations have implemented measures to move in the direction of accomplishing UHC, but it has been quite variable. Low and middle income nations find it hard to make considerable growth due to the varied health needs of the general population and the extent of financial investment required in that regard. The need of the hour is to strengthen the health care delivery system, which will essentially require infrastructure support and an adequate number of the health workforce to bring the desired change.

In addition, there is a need to ensure capacity building through training of health workers, involvement of all the stakeholders, including international welfare agencies \& non-governmental organizations and a multisectoral, yet an integrated approach. Simultaneously, there is an immense need to strengthen primary health care and ensure that all the health care services are integrated with the primary health care, which will ensure the availability of services to those who need them the most. Further, special attention should be given towards the research front, in order to improvise and develop newer modes of diagnostics, therapeutic modalities and development of vaccines.

Also, it is very essential to strengthen health information system, surveillance mechanisms, expand advocacy, communication and social mobilization activities targeting all the public health priorities in a tailor-made manner. However, it is quite obvious that none of these can be sustainable unless there is a strong political commitment and financial support towards the health sector for the betterment of the health indices. Finally, the mere implementation of strategies to accelerate the accomplishment of UHC won't serve the purpose, unless there is a mechanism to monitor the progress..

To conclude, universal health coverage goes way beyond health alone and any measures taken to achieve the same indirectly denote the steps taken for the attainment of equity and social inclusion. It is the responsibility of all the stakeholders to work in a concerted and coordinated manner to accomplish universal health coverage by 2030 .

\author{
Dr. Saurabh RamBihariLal Shrivastava \\ Vice-Principal Curriculum, \\ FAIMER Fellow 2017 \\ Kancheepuram District, Tamil Nadu, India
}

Associate Professor, Department of Community Medicine, Shri Sathya Sai Medical College \& Research Institute, Sri Balaji Vidyapeeth - Deemed to be University, Ammapettai, Nellikuppam, Chengalpet Taluk, 